\title{
Comprehensive Review of the Cardinal Ligament
}

\author{
Seif Eid ${ }^{1}$, Joe Iwanaga ${ }^{2}$, Rod J. Oskouian ${ }^{3}$, Marios Loukas ${ }^{1}$, R. Shane Tubbs ${ }^{4}$ \\ 1. Anatomical Sciences, St. George's University, St. George's, GRD 2. Seattle Science Foundation, Seattle, USA 3. \\ Neurosurgery, Swedish Neuroscience Institute, Seattle, USA 4. Neurosurgery, Seattle Science Foundation, Seattle, USA
}

Corresponding author: Joe Iwanaga, iwanagajoeca@gmail.com

\section{Abstract}

The cardinal ligament is thought to be one of the important structures in providing support and stabilization for the pelvic organs e.g., the uterus. However, many discrepancies exist in the literature regarding terminology, anatomy, and histology. The cardinal ligament attaches the lateral side of the vagina and cervix to the lateral pelvic wall, which provides support to the vagina and cervix. Studies have shown variable findings in the collagen content and distribution in the cardinal ligaments of women with a prolapsed uterus. Uterine vessels and the branches of the inferior hypogastric plexus travel in the cardinal ligament, which is of great importance during pelvic surgeries. Cervical cancer may lead to metastatic spread to the lymph nodes juxtaposed to the cardinal ligaments. This review aims to highlight the clinical significance and surgical importance of the cardinal ligament with a comparison with previous studies.

Categories: Obstetrics/Gynecology

Keywords: cardinal ligament, transverse cervical ligament, pelvic prolapse, uterus, parametrium

\section{Introduction And Background}

The support of the pelvic organs is contributed to by the cardinal, uterosacral, and uterovesicular ligaments and the pelvic floor musculature. Of these, known by several names, the cardinal ligament (Figure 1) is a structure that provides the support and stabilization of the cervix and the upper vagina to the pelvic wall. It is important to note that the cardinal ligament is not a skeletal ligament and contains arteries, veins, and nerves. Various inconsistencies appear in the literature regarding this structure due to the variability and complexity of pelvic pathology. This review aims to highlight the anatomical and clinical significance of the cardinal ligament to provide a better understanding for clinicians.

Received 06/11/2018

Review began 06/18/2018 Review ended 06/18/2018 Published 06/20/2018

(c) Copyright 2018

Eid et al. This is an open access article distributed under the terms of the Creative Commons Attribution License CC-BY 3.0., which permits unrestricted use, distribution, and reproduction in any medium, provided the original author and source are credited.

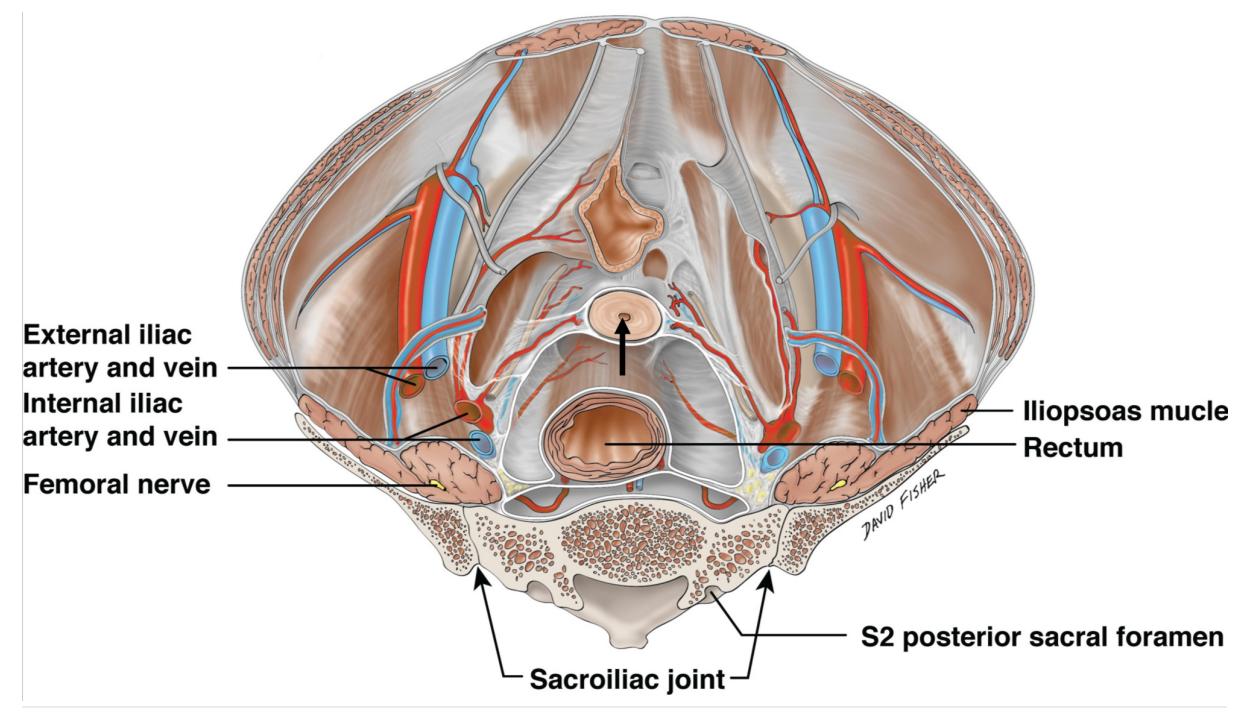

FIGURE 1: Superior view of the female pelvis external to the peritoneum

Note the lower uterus cut in cross-section (arrow) and being suspended laterally by the cardinal ligaments carrying the uterine arteries. Also note the ureters just inferior to the uterine arteries and their relationship to the cardinal ligaments.

Review

History

In 1880, Josef Kocks was the first to name this structure the cardinal ligament [1]. Later, in 1895, Mackenrodt 
named it the transverse cervical ligament and since then, it often takes on the eponym, Mackenrodt's ligament [2]. The literature contains different terminologies and descriptions of the cardinal ligament [3], although the most recent Terminologia Anatomica term for this structure is the cardinal ligament. Interestingly, some have even questioned the existence of the cardinal ligament as a separate entity. For example, Moritz reported that this ligament is a continuation of the parametrium, which can be separated only artificially by dissection [4].

\section{Anatomy}

Although several differences exist regarding the identification of the cardinal ligament, the functional ability of this ligament to provide pelvic support is agreed upon. The cardinal ligaments are not skeletal ligaments composed of regular dense connective tissue, rather these are mesenteric structures consisting of loose connective tissue and smooth muscle [5]. Range and Woodburne [6] described the cardinal ligament as a mesenteric condensation composed of loose areolar connective tissue surrounded by blood vessels, nerves, and lymphatics, which can only be visually distinguished when the uterus is retracted to the contralateral side. The cardinal ligaments originate from the lateral pelvic wall and attach on the lateral cervix and vagina. These ligaments collaborate with the uterosacral ligaments and the pelvic musculature to provide support to the pelvic organs and to prevent prolapse. The cardinal ligament is vertically oriented while the uterosacral ligaments are dorsally oriented in the standing position, which collectively provides apical support for the uterus and vagina [7]. As the uterine vessels and inferiorly located ureter are related to the cardinal ligament, they can be injured during pelvic surgery where the cardinal ligament is manipulated. The ureter is approximately $2 \mathrm{~cm}$ lateral to the supravaginal region of the cervix [5]. Fibers from the uterovaginal plexus derived from the inferior hypogastric plexus travel in the cardinal ligament, which provides innervation to the clitoris, vestibular glands, and vaginal wall [8]. A cadaveric study revealed that the cardinal ligament was continuous with the vesicohypogastric fascia and that the uterine artery can be found in the superior region of the cardinal ligament while the uterine vein or middle vesical artery/vein can be found in its inferior region [9]. Magnetic resonance imaging (MRI) has aided in constructing 3D models that demarcated the cardinal and uterosacral ligaments and that demonstrated that the cardinal ligament is longer and more curved than the latter [7].

\section{Histology}

The cardinal ligament can be subdivided based on histological characteristics. Range and Woodburne [6] revealed variable arrangement in collagen fibers and inconsistent cellular and vascular components in different regions of the cardinal ligament on microscopic examination. Another histologic study revealed increased expression of collagen III and tenascin with decreased elastin in the cardinal ligaments of women with a prolapsed uterus [10]. On the other hand, some studies have reported decreased levels of collagen in women with pelvic organ prolapse and urinary incontinence [11-13]. Salman et al. [14] found that the collagen fibers were more densely arranged and were thicker in women without uterine prolapse.

Kato et al. [15] revealed the presence of branches of the pelvic splanchnic nerves in the dorsal aspect of the cardinal ligament, which was separated by the vascular portion by loose connective tissue. Some researchers were able to demonstrate nerve content by immunofluorescence techniques and found that the cardinal ligament consisted of many free nerve fibers with a significant concentration of these fibers in the lateral third of the ligament [16]. Another study of patients with cervical cancer discovered an association between high-risk disease and the presence of metastatic disease in the lymphatics found in the vascular region of the cardinal ligament along with pelvic lymph nodes [17]. This would suggest that the complete removal of the vascular portion of the cardinal ligament might be indicated.

\section{Clinical associations}

Although many disagreements exist regarding the terminology, the clinical implications of the cardinal ligament related to disorders of the pelvic floor and cervical malignancy must be acknowledged. It is important to note that the pelvic floor musculature and the pelvic fascia mutually contribute to the stability of the pelvic floor [18]. The levator ani muscle provides support to the pelvic organs and consists of the puborectalis, pubococcygeus, and iliococcygeus muscles [19]. In a study using 3D models from MRI, authors have developed four patterns of levator ani avulsion pathology that was associated with pelvic organ prolapse. This study also revealed that there was a significant difference in the morphology of the puborectalis and pubococcygeus parameters in women with prolapse compared to the control group while there was no difference in the morphology of the iliococcygeus muscle [19]. Otcenasek et al. [18] performed a similar study to classify the causes of pelvic prolapse and revealed that prolapse may occur due to a defect of the fascia and can be either partial or complete tears while the muscle defects can be variable. Some authors have identified the cardinal ligament as a structure consisting of the lateral parametrium observed from the dissected paravesical space while the transverse cervical ligament is observed from the dissected pararectal space [9]. Yabuki describes the cardinal ligament and the transverse cervical ligament as two different structures that comprise the supporting system of the pelvis [20]. He emphasizes that the cardinal ligament represents only the medial side of the supporting system while the transverse cervical ligament is the lateral continuation of the cardinal ligament that forms a complex with the lateral ligament of the rectum and the umbilical ligament [20]. 
From a surgical perspective, the cardinal ligament still remains a vital landmark, as it is one of the pedicles to be secured during a hysterectomy. According to Cruikshank and Kovac [21], the traction and cutting of the cardinal ligament are the main factors that affect ureter movement during vaginal hysterectomy. This leads to the movement of the ureter out of the operative field, thereby protecting the ureter from possible injury. One recent study evaluating different guideline protocols proposed that the possibility of pelvic prolapse after hysterectomy may decrease by the suspension of the vaginal vault to the cardinal and uterosacral ligaments [22]. Another important surgical consideration is the prediction of the uterine anatomy based on the cervical position, which is an important consideration for several gynecological procedures. Fidan et al. [23] revealed that the anatomy of the uterus and adjacent tissues can be predicted by pelvic examination and confirmed with ultrasonography. This study revealed that the uterus was anteverted in $90 \%$ of cases with the cervix being in the posterior fornix position while uterine anteversion was present in $64.2 \%$ of cases with an anterior cervix [23].

In addition, the cardinal ligament is also involved in radical hysterectomy for cervical cancer due to the lymphatic drainage of the cervix. Girardi et al. [24] revealed that both positive and negative pelvic nodes can be found in variable locations along the parametrium, even at the most lateral region near the pelvic side wall. This study also revealed that positive parametrial lymph nodes are strongly associated with the presence of positive pelvic lymph nodes and a higher recurrence rate of cervical cancer [24]. This may suggest more extensive resection of the cardinal ligament to ensure a disease-free state. However, one must also consider possible damage to the pelvic plexuses traveling in these ligaments to avoid urinary bladder, rectal, and sexual complications that may arise when these ligaments are resected during a radical hysterectomy [25]. It was previously reported that the nerve content is variable along the length of the cardinal ligament; however, there is an increased nerve content in its lateral thirds from the pelvic wall [16].

Ramanah et al. [26] analyzed the literature and describes the cardinal and uterosacral ligaments as "visceral ligaments," which contain blood vessels, nerves, connective tissue, and adipose tissue. These authors described the origin of the internal iliac artery as the proximal insertion point and the cervix and upper vagina as the distal insertion point of the cardinal ligament [26]. Another review also revealed similar findings and inconsistencies relating to the description and characteristics of the cardinal ligament [27]. This signifies the importance of obtaining a consistent description of these ligaments in order to better understand the pathologies of the pelvic floor.

\section{Conclusions}

The aim of this review was to highlight some of the disagreements and to provide a better understanding of the nature of the cardinal ligament. Due to the complexity of the pelvic floor, further research is warranted to clear some of the inconsistencies in the literature. Despite the differences in terminology, the clinical and surgical significance of this ligament is paramount in providing ideal patient care.

\section{Additional Information}

\section{Disclosures}

Conflicts of interest: In compliance with the ICMJE uniform disclosure form, all authors declare the following: Payment/services info: All authors have declared that no financial support was received from any organization for the submitted work. Financial relationships: All authors have declared that they have no financial relationships at present or within the previous three years with any organizations that might have an interest in the submitted work. Other relationships: All authors have declared that there are no other relationships or activities that could appear to have influenced the submitted work.

\section{References}

1. Kocks J: Die Normale und Patologhische Lage und Gestalt des Uterus sowie deren Mechanik [Book in German]. Verlag von Max Cohen, Bonn; 1880.

2. Mackenrodt A: Ueber die Ursachen der normalen und pathologischen Lagen des Uterus [Article in German] . Arch Gynecol Obstet. 1895, 48:393-421. 10.1007/BF01979925

3. Ercoli A, Delmas V, Fanfani F, et al.: Terminologia Anatomica versus unofficial descriptions and nomenclature of the fasciae and ligaments of the female pelvis: a dissection-based comparative study. Am J Obstet Gynecol. 2005, 193:1565-1573. 10.1016/j.ajog.2005.05.007

4. Moritz M: On the nature of the so-called ligaments of Mackenrodt . BJOG. 1913, 23:135-138. 10.1111/j.14710528.1913.tb12756.x

5. Standring S: Gray's Anatomy: The Anatomical Basis of Clinical Practice. Elsevier Limited, Philadelphia; 2016. 41st edition:1199-1204.

6. Range RL, Woodburne RT: The gross and microscopic anatomy of the transverse cervical ligament . Am J Obstet Gynecol. 1964, 90:460-467. 10.1016/0002-9378(64)90802-6

7. Chen L, Ramanah R, Hsu Y, Ashton-Miller JA, Delancey JO: Cardinal and deep uterosacral ligament lines of action: MRI based 3D technique development and preliminary findings in normal women. Int Urogynecol J. 2012, 24:37-45. 10.1007/s00192-012-1801-4

8. Shoja MM, Sharma A, Mirzayan N, Groat C, Watanabe K, Loukas M, Tubbs RS: Neuroanatomy of the female abdominopelvic region: a review with application to pelvic pain syndromes. Clin Anat. 2012, 26:66-76. $10.1002 /$ ca.22200 
9. Yabuki Y, Sasaki H, Hatakeyama N, Murakami G: Discrepancies between classic anatomy and modern gynecologic surgery on pelvic connective tissue structure: harmonization of those concepts by collaborative cadaver dissection. Am J Obstet Gynecol. 2005, 193:7-15. 10.1016/j.ajog.2005.02.108

10. Ewies AA, Al-Azzawi F, Thompson J: Changes in extracellular matrix proteins in the cardinal ligaments of post-menopausal women with or without prolapse: a computerized immunohistomorphometric analysis. Hum Reprod. 2003, 18:2189-2195. 10.1093/humrep/deg420

11. Iwahashi M, Muragaki Y: Decreased type III collagen expression in human uterine cervix of prolapse uteri . Exp Ther Med. 2011, 2:271-274. 10.3892/etm.2011.204

12. Takano CC, Giaro MJ, Sartori MG, et al.: Analysis of collagen in parametrium and vaginal apex of women with and without uterine prolapse. Int Urogynecol J Pelvic Floor Dysfunct. 2002, 13:342-345. 10.1007/s001920200076

13. Chen BH, Wen Y, Li H, Polan ML: Collagen metabolism and turnover in women with stress urinary incontinence pelvic prolapse. Int Urogynecol J Pelvic Floor Dysfunct. 2002, 13:80-87. 10.1007/s001920200020

14. Salman MC, Ozyuncu O, Sargon MF, Kucukali T, Durukan T: Light and electron microscopic evaluation of cardinal ligaments in women with or without uterine prolapse. Int Urogynecol J. 2010, 21:235-239. 10.1007/s00192-009-1006-7

15. Kato T, Murakami G, Yabuki Y: Does the cardinal ligament of the uterus contain a nerve that should be preserved in radical hysterectomy?. Anat Sci Int. 2002, 77:161-168. 10.1046/j.0022-7722.2002.00023.x

16. Butler-Manuel SA, Buttery LD, Ahern RP, Polak JM, Barton DP: Pelvic nerve plexus trauma at radical hysterectomy and simple hysterectomy. Cancer. 2000, 89:834-841.

17. Hoffman MS, Williams V, Salihu HM, Gunasekaran S, Sayer RA, Hakam A, Roberts WS: The vascular portion of the cardinal ligament: surgical significance during radical hysterectomy for cervical cancer. Am J Obstet Gynecol. 2008, 199:191. 10.1016/j.ajog.2008.04.030

18. Otcenasek M, Gauruder-Burmester A, Haak LA, Grill R, Popken G, Baca V: Paravaginal defect: a new classification of fascial and muscle tears in the paravaginal region. Clin Anat. 2016, 29:524-529. 10.1002/ca.22694

19. Li R, Song Y, Ma M: Relationship between levator ani and bony pelvis morphology and clinical grade prolapse in women. Clin Anat. 2016, 28:813-819. 10.1002/ca.22536

20. Yabuki Y: Clinical anatomy of the subserous layer: an amalgamation of gross and clinical anatomy . Clin Anat. 2016, 29:508-515. 10.1002/ca.22579

21. Cruikshank SH, Kovac SR: Role of the uterosacral-cardinal ligament complex in protecting the ureter during vaginal hysterectomy. Int J Gynaecol Obstet. 1993, 40:141-144. 10.1016/0020-7292(93)90374-6

22. Sloth SB, Schroll JB, Settnes A, et al.: Systematic review of the limited evidence for different surgical techniques at benign hysterectomy: A clinical guideline initiated by the Danish health authority. Eur J Obstet Gynecol Reprod Biol. 2017, 216:169-177. 10.1016/j.ejogrb.2017.07.012

23. Fidan I, Keskin U, Ulubay M, Öztürk M, Bodur S: Value of vaginal cervical position in estimating uterine anatomy. Clin Anat. 2017, 30:404-408. 10.1002/ca.22854

24. Girardi F, Lichtenegger W, Tamussino K, Haas J: The importance of parametrial lymph nodes in the treatment of cervical cancer. Gynecol Onc. 1989, 34:206-211. 10.1016/0090-8258(89)90143-1

25. Butler-Manuel SA: Self-assessment of morbidity following radical hysterectomy for cervical cancer . J Obstet Gynaecol. 1999, 19:180-183. 10.1080/01443619965552

26. Ramanah R, Berger MB, Paratte BM, Delancey JO: Anatomy and histology of apical support: a literature review concerning cardinal and uterosacral ligaments. Int Urogynecol J. 2012, 23:1483-1494. 10.1007/s00192-012-1819-7

27. Iancu G, Doumouchtsis SK: A historical perspective and evolution of our knowledge on the cardinal ligament. Neurourol Urodyn. 2014, 33:380-386. 10.1002/nau.22421 